\title{
Floristic Composition and Natural History Characteristics of Dry Forests in the Pacific ${ }^{1}$
}

\author{
Thomas W. Gillespie, ${ }^{2,3}$ Gunnar Keppel, ${ }^{4}$ Stephanie Pau ${ }^{2,5}$ Jonathan P. Price, ${ }^{6}$ Tanguy Faffré, ${ }^{7}$ \\ Jean-Yves Meyer, and Kristin O'Neill ${ }^{2,9}$
}

\begin{abstract}
We compare the floristic composition of tropical dry forests at the stand level using Gentry's transect method (0.1 ha) in some of the largest and highest-quality remaining fragments in the Pacific (Hawai' $i, 15$ sites; Fiji, 9; the Marianas, 3; the Marquesas, 6; New Caledonia, 7) and compare results with neotropical dry forests. A total of 299 species or morphospecies $\geq 2.5 \mathrm{~cm}$ diameter at breast height were identified from all 40 sites in the Pacific. Rubiaceae (28 spp.), Euphorbiaceae (25 spp.), Fabaceae (23 spp.), Sapindaceae (18 spp.), and Myrtaceae (17 spp.) were the most speciose families in Pacific dry forest; however, no family dominated across regions in the Pacific. The most common species by frequency and density in each region were native with the exception of Hawai $i$, which contains a high number of nonnative species. Observed and estimated (Chao 2) levels of native species richness show that New Caledonia and Fiji contain the highest species richness followed by Hawai'i, the Marianas, and the Marquesas. There is very little overlap at the native species level among regions, with Hawaiian dry forests the most dissimilar at the native species, genus, and family level and New Caledonia and Fiji the most similar. Unlike mainland neotropical dry forest, dry forests in the Pacific contain very few deciduous species and a low proportion of wind-dispersed species. There is a high proportion of dioecious species in Hawai'i, which is similar to the neotropics; however, other Pacific regions have fewer dioecious species.
\end{abstract}

The tropical Pacific harbors several biodiversity hot spots, with high levels of restricted-range and endemic species (Myers et al. 2000). Despite this unique flora and fauna, forests of the tropical Pacific have been little studied compared with those of other tropical regions (Keppel 2006, Keppel et al.

\footnotetext{
${ }^{1}$ This research was funded by the National Geographic Society, National Science Foundation BCS0455052, Five Undergraduate Research Fellowship, UCLA Department of Geography, UCLA Stephen A. Varva Fellowship, NASA Earth System Science Fellowship, and the UCLA Dissertation Year Fellowship. Manuscript accepted 20 June 2010.

2 Department of Geography, University of California Los Angeles, Los Angeles, California 90095-1524 (fax: 310-206-5976).

${ }^{3}$ Corresponding author (e-mail: tg@geog.ucla.edu).
}

Pacific Science (2011), vol. 65, no. 2:127-141

doi: $10.2984 / 65.2 .127$

(C) 2011 by University of Hawai'i Press

All rights reserved
2010). This is especially true for tropical dry forests, which are among the most threatened ecosystems worldwide (Janzen 1988, Lerdau et al. 1991) and in the Pacific (Gillespie and Jaffré 2003, Keppel and Tuiwawa 2007, Meyer 2007, Pau et al. 2009). New Caledonia, Fiji, the Marquesas, the Marianas, and the

\footnotetext{
${ }^{4}$ Curtin Biodiversity and Climate Institute, Curtin University of Technology, GPO Box U1987, Perth, 6845 Western Australia, Australia (e-mail: G.Keppel@curtin. edu.au).

${ }^{5}$ e-mail: paus@ucla.edu.

${ }^{6}$ Department of Geography and Environmental Studies, University of Hawai'i at Hilo, Hilo, Hawai'i 96720 (e-mail: jpprice@hawaii.edu).

7 Institut de Recherche pour le Développement (IRD), UMR AMAP, Laboratoire de Botanique et d'Ecologie Végétale Appliquées, Herbarium NOU, 98848, Nouméa, New Caledonia (e-mail: tanguy.jaffre@ ird.fr).

${ }^{8}$ Délégation à la Recherche Ministère de l'Education, de l'Enseignement Supérieur et de la Recherche, Gouvernement de Polynésie française, B.P. 20981, Papeete, Tahiti (e-mail: jean-yves.meyer@recherche.gov.pf).

${ }^{9}$ e-mail: kristinoneill@ucla.edu.
} 
Hawaiian Islands once contained large expanses of native forests on the dry side of islands. However, this forest type has experienced a considerable loss of habitat, and dry forests in the Pacific have been identified as critically endangered, with less than $5 \%$ of their former extent remaining (Janzen 1988, Myers et al. 2000, Rolett and Diamond 2004).

Quantitative plot data could provide information about similarities and differences in the diversity and composition at various taxonomic levels (e.g., family, genus, and species) for remaining stands of dry forests in each Pacific region. Currently, there are little quantitative plot data for dry forests in the region. New Caledonia (71 sites) and Fiji (10 sites) have extensive qualitative data (species presence/absence) for tropical dry forests; however, there are little comparative plot data (Harlow and Biciloa 2001, Gillespie and Jaffré 2003, Keppel and Tuiwawa 2007, Jaffré et al. 2008). Similarly, the tropical dry forests of the Marianas, the Marquesas, and the Hawaiian islands have qualitative data for several islands but little or no comparative quantitative plot data (Hatheway 1952, Craig 1992, Wagner and Lorence 2003).

Gentry (1995) undertook the largest analysis of tropical dry forest sites in the world, and this has provided valuable information on geographical patterns of floristic composition and natural history characteristics within neotropical dry forests. That analysis concluded that Fabaceae is consistently the dominant family of trees in 0.1 ha areas in neotropical sites, Bignoniaceae is consistently the dominant family of lianas in dry forests, and Myrtaceae, Rubiaceae, Sapindaceae, Euphorbiaceae, and Flacourtiaceae are the other most speciose woody families in the neotropical dry forest (Gentry 1995). Gentry (1995) also noted that tropical dry forests in the neotropics have a high proportion of wind-dispersed species and a high percentage of deciduous species when compared with moist forests. However, recent analyses of tropical dry forests on islands in the Caribbean have noted that these tropical dry forests have different natural history characteristics of dispersal types and canopy phenology than those of tropical dry forests in the mainland neotropics, although the proportions of sexual systems are similar (Gillespie 2006, Lugo et al. 2006). There are also an increasing number of studies that suggest that the natural history characteristics of dry forests on Pacific islands should have a higher incidence of dioecious species, fewer wind-dispersed species, and be dominated by evergreen trees (Keppel and Tuiwawa 2007, Whittaker and Fernández-Palacios 2007, Pau et al. 2009).

Our research on the native dry forests of the Pacific has three primary objectives. First, we report on family and species composition of tropical dry forests within the Pacific region (New Caledonia, Fiji, the Marquesas, the Marianas, Hawai'i). Second, we examine native species richness and the floristic similarities of native families, genera, and species within dry forest regions in the Pacific. Third, we examine natural history characteristics of native species in tropical dry forests. In particular, we test the hypothesis that dry forests in the Pacific contain a high proportion of evergreen species, a high proportion of dioecious species, and a low proportion of winddispersed species.

\section{MATERIALS AND METHODS}

\section{Study Sites}

Tropical dry forest can be loosely defined as forest in frost-free regions with 500-2,000 $\mathrm{mm}$ of annual precipitation and a pronounced dry season of 4 to 7 months (Holdridge et al. 1971, Walter 1971, Murphy and Lugo 1986). Field research was conducted from 2001 to 2006 in five regions (New Caledonia, 7 sites; Fiji, 10; the Marquesas, 6; the Marianas, 3; Hawai $i$, 15) that contain tropical dry forest (Figure 1). Study sites were selected by examining annual precipitation from WorldClim (Hijmans et al. 2005), Landsat ETM+, and Shuttle Radar Topography imagery from 2001 to identify the largest forest fragments that occur on the dry side in each region. Potential sites were then discussed with local botanists and surveyed in the field to ensure that they were dominated by native species. We selected sites that had been disturbed (e.g., fires, wood collection, grazing) as little as possible based on available information. 

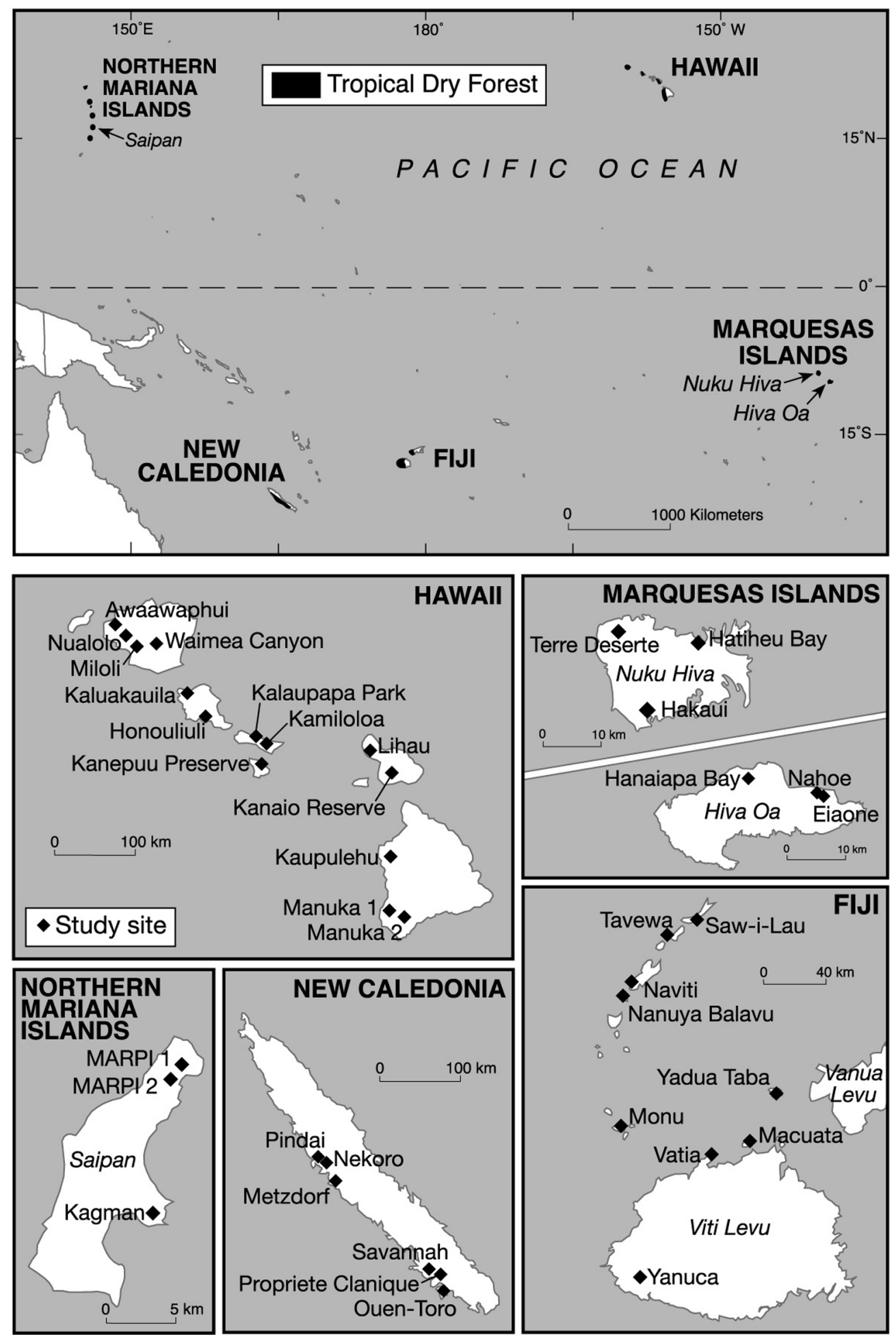

Figure 1. Tropical dry forests in the Pacific and locations of dry forest sites in each region. 
The sites represent the largest and highestquality remaining patches of tropical dry forest known on islands in each region.

Dry forest study sites in the Pacific were located at low elevations $(<200 \mathrm{~m})$ with the exception of dry forest in Hawai'i, where remaining sites were located between 515 and $1,014 \mathrm{~m}$ (Table 1). Native forest on the dry side of each region in the Pacific varied in mean annual precipitation and precipitation seasonality (coefficient of variation of monthly precipitation) according to WordClim. New Caledonia (range 1,010 to $1,360 \mathrm{~mm}$ ) and the Marquesas (1,003 to $1,428 \mathrm{~mm}$ ) received low annual precipitation and had low precipitation seasonality, but the Marianas (1,990 to 2,029 $\mathrm{mm})$ and Fiji $(1,763$ to 2,289 $\mathrm{mm})$ had higher levels of annual precipitation and higher precipitation seasonality. Hawai'i contained the largest variation in annual precipitation and precipitation seasonality among all regions. Study sites were primarily on volcanic substrates in Fiji, Hawai'i, and the Marquesas. The Marianas study sites were located on limestone substrates, and there were a diversity of substrates in New Caledonia.

\section{Field Methods}

Woody plants were quantified at the stand level in tropical dry forests of the Pacific. Data on floristic composition at each study site followed Gentry $(1982,1988)$. Belt transects totaling $1,000 \mathrm{~m}^{2}$ or 0.1 ha were established at each site. Each sample consisted of 10 transects $(2 \mathrm{~m} \times 50 \mathrm{~m})$ spaced $10 \mathrm{~m}$ apart in which the diameter at breast height (dbh) of all plants with $\mathrm{dbh} \geq 2.5 \mathrm{~cm}$ rooted in the sample area was recorded (Gentry 1988). Transects were located in mature stands or stands judged to be in an advanced state of regeneration following Gentry (1988) and were established $30 \mathrm{~m}$ from the edge where possible.

Plants encountered in the transects from New Caledonia, Fiji, and Hawai'i were identified to species or morphospecies at the local herbaria: Institut de Recherche pour le Développement (IRD) in Nouméa, New Caledonia; South Pacific Regional Herbarium (SUVA) at the University of the South Pacific in Suva, Fiji; and Bishop Museum Herbarium Pacifi- cum (BISH) in Honolulu, Hawai'i. All species encountered in transects in the Marianas were identified using Stone (1970), and all species from the Marquesas were identified using Wagner and Lorence (2003). Family taxonomy for all sites follows Wagner et al. (1990) and Gentry (1995).

\section{Natural History Characteristics}

Natural history characteristics of all native woody plants encountered in transects were classified by leaf phenology, sexual system, and dispersal types based on available literature (Stone 1970, Wagner et al. 1990, Wagner and Lorence 2003, Keppel and Tuiwawa 2007), examination of herbarium specimens, and cumulative field experience of local botanists. Plant leaf phenology was classified as evergreen, semideciduous, or deciduous. Species were classified into three sexual system categories: hermaphrodite, monoecious, and dioecious following Bawa et al. (1985). The perfect category (bisexual flowers) included all monostylous and heterostylous hermaphroditic species. The monoecious category (male and female flowers on the same plant) included all andromonoecious, polygamomonoecious, and strictly monoecious species. The dioecious category (male and female flowers on different plants) included all androdioecious, gynodioecious, and strictly dioecious species. Dispersal types were classified into three categories based on fruit and seed types: anemochory, autochory, and zoochory. Anemochoric species are winddispersed plants that generally have winged or plumose seeds. Autochoric species are plants that have explosive pods or capsules that usually disperse the seed. Zoochoric species are vertebrate-dispersed plants with a fleshy exocarp.

\section{Data Analysis}

Total family richness was calculated for all species encountered in each region in the $\mathrm{Pa}$ cific. All species were ranked by frequency and density to identify the most common species in each region. Native species richness estimates for each region were assessed based 
TABLE 1

Study Site Location, Elevation, Annual Precipitation (Ppt), and Precipitation Seasonality (Seas) from Hijmans et al. (2005), and Geologic Substrate

\begin{tabular}{|c|c|c|c|c|c|c|}
\hline Study Sites (Code) & Latitude & Longitude & Elevation (m) & Ppt (mm) & Seas & Substrate \\
\hline \multicolumn{7}{|l|}{ New Caledonia } \\
\hline Ouen Toro 1 (N1) & $22^{\circ} 18^{\prime} 38.12^{\prime \prime} \mathrm{S}$ & $166^{\circ} 27^{\prime} 16.80^{\prime \prime} \mathrm{E}$ & 37 & 1,010 & 34 & Sandstone \\
\hline Ouen Toro 2 (N2) & $22^{\circ} 18^{\prime} 31.54^{\prime \prime} \mathrm{S}$ & $166^{\circ} 27^{\prime} 13.24^{\prime \prime} \mathrm{E}$ & 54 & 1,010 & 34 & Sandstone \\
\hline Pindai (N3) & $21^{\circ} 20^{\prime} 5.47^{\prime \prime} \mathrm{S}$ & $164^{\circ} 58^{\prime} 11.99^{\prime \prime} \mathrm{E}$ & 36 & 1,270 & 47 & Limestone \\
\hline Clanique (N4) & $22^{\circ} 11^{\prime} 45.74^{\prime \prime} \mathrm{S}$ & $166^{\circ} 27^{\prime} 39.07^{\prime \prime} \mathrm{E}$ & 160 & 1,292 & 34 & Volcanic \\
\hline Savannah (N5) & $22^{\circ} 9^{\prime} 15.13^{\prime \prime} \mathrm{S}$ & $166^{\circ} 23^{\prime} 36.11^{\prime \prime} \mathrm{E}$ & 129 & 1,079 & 35 & Volcanic \\
\hline Metzdorf (N6) & $21^{\circ} 25^{\prime} 26.12^{\prime \prime} \mathrm{S}$ & $165^{\circ} 8^{\prime} 47.14^{\prime \prime} \mathrm{E}$ & 13 & 1,322 & 47 & Alluvial \\
\hline Nekoro (N7) & $21^{\circ} 19^{\prime} 57.22^{\prime \prime} \mathrm{S}$ & $165^{\circ} 2^{\prime} 34.45^{\prime \prime} \mathrm{E}$ & 16 & 1,360 & 47 & Limestone \\
\hline \multicolumn{7}{|l|}{ Fiji } \\
\hline Yanuca $(\mathrm{F} 1)$ & $18^{\circ} 8^{\prime} 56.98^{\prime \prime} \mathrm{S}$ & $177^{\circ} 26^{\prime} 16.80^{\prime \prime} \mathrm{E}$ & 19 & 2,087 & 50 & Limestone \\
\hline Vatia (F2) & $17^{\circ} 23^{\prime} 24.19^{\prime \prime} \mathrm{S}$ & $177^{\circ} 48^{\prime} 47.95^{\prime \prime} \mathrm{E}$ & 81 & 2,117 & 52 & Volcanic \\
\hline Monu (F3) & $17^{\circ} 35^{\prime} 54.98^{\prime \prime} \mathrm{S}$ & $177^{\circ} 2^{\prime} 38.17^{\prime \prime} \mathrm{E}$ & 42 & 1,795 & 62 & Volcanic \\
\hline Naviti (F4) & $17^{\circ} 9^{\prime} 16.67^{\prime \prime} \mathrm{S}$ & $177^{\circ} 11^{\prime} 37.16^{\prime \prime} \mathrm{E}$ & 56 & 1,777 & 61 & Volcanic \\
\hline Sawa-I-Lau (F5) & $16^{\circ} 50^{\prime} 58.95^{\prime \prime} \mathrm{S}$ & $177^{\circ} 28^{\prime} 6.80^{\prime \prime} \mathrm{E}$ & 21 & 1,762 & 60 & Limestone \\
\hline Nanuya Balavu (F6) & $17^{\circ} 10^{\prime} 36.43^{\prime \prime} \mathrm{S}$ & $177^{\circ} 10^{\prime} 57.19^{\prime \prime} \mathrm{E}$ & 34 & 1,777 & 61 & Volcanic \\
\hline Tavewa (F7) & $16^{\circ} 55^{\prime} 21.23^{\prime \prime} \mathrm{S}$ & $177^{\circ} 21^{\prime} 46.42^{\prime \prime} \mathrm{E}$ & 61 & 1,763 & 61 & Volcanic \\
\hline Yadua Taba (F8) & $16^{\circ} 50^{\prime} 18.20^{\prime \prime} \mathrm{S}$ & $178^{\circ} 16^{\prime} 45.34^{\prime \prime} \mathrm{E}$ & 69 & 2,285 & 49 & Volcanic \\
\hline Macuata (F9) & $17^{\circ} 21^{\prime} 14.90^{\prime \prime} \mathrm{S}$ & $178^{\circ} 1^{\prime} 55.28^{\prime \prime} \mathrm{E}$ & 32 & 2,289 & 46 & Volcanic \\
\hline \multicolumn{7}{|l|}{ Marquesas } \\
\hline Hatiheu Bay (Q1) & $8^{\circ} 49^{\prime} 30.16^{\prime \prime} \mathrm{S}$ & $140^{\circ} 5^{\prime} 31.14^{\prime \prime} \mathrm{W}$ & 84 & 1,086 & 36 & Volcanic \\
\hline Hakaui (Q2) & $8^{\circ} 55^{\prime} 47.29^{\prime \prime} \mathrm{S}$ & $140^{\circ} 10^{\prime} 27.34^{\prime \prime} \mathrm{W}$ & 126 & 1,003 & 37 & Volcanic \\
\hline Nahoe (Q3) & $9^{\circ} 44^{\prime} 35.96^{\prime \prime} \mathrm{S}$ & $138^{\circ} 54^{\prime} 51.95^{\prime \prime} W$ & 180 & 1,404 & 27 & Volcanic \\
\hline Hanaiapa Bay (Q4) & $9^{\circ} 43^{\prime} 9.73^{\prime \prime} \mathrm{S}$ & $139^{\circ} 1^{\prime} 9.17^{\prime \prime} \mathrm{W}$ & 151 & 1,428 & 26 & Volcanic \\
\hline Eiaone (Q5) & $9^{\circ} 44^{\prime} 35.43^{\prime \prime} \mathrm{S}$ & $138^{\circ} 54^{\prime} 28.41^{\prime \prime} \mathrm{W}$ & 148 & 1,405 & 26 & Volcanic \\
\hline Terre Déserte (Q6) & $8^{\circ} 48^{\prime} 29.85^{\prime \prime} \mathrm{S}$ & $140^{\circ} 12^{\prime} 43.12^{\prime \prime} \mathrm{W}$ & 221 & 1,186 & 34 & Volcanic \\
\hline \multicolumn{7}{|l|}{ Marianas } \\
\hline MARPI 1 (M1) & $15^{\circ} 15^{\prime} 7.00^{\prime \prime} \mathrm{N}$ & $145^{\circ} 47^{\prime} 56.92^{\prime \prime} \mathrm{E}$ & 201 & 2,029 & 51 & Limestone \\
\hline MARPI 2 (M2) & $15^{\circ} 15^{\prime} 19.47^{\prime \prime} \mathrm{N}$ & $145^{\circ} 48^{\prime} 20.70^{\prime \prime} \mathrm{E}$ & 123 & 1,990 & 52 & Limestone \\
\hline Kargman (M3) & $15^{\circ} 9^{\prime} 43.43^{\prime \prime} \mathrm{N}$ & $145^{\circ} 47^{\prime} 23.86^{\prime \prime} \mathrm{E}$ & 63 & 1,998 & 52 & Limestone \\
\hline \multicolumn{7}{|l|}{ Hawai'i } \\
\hline Manukā 1 (H1) & $19^{\circ} 6^{\prime} 49.50^{\prime \prime} \mathrm{N}$ & $155^{\circ} 48^{\prime} 53.43^{\prime \prime} \mathrm{W}$ & 662 & 1,318 & 12 & Volcanic \\
\hline Manukā 2 (H2) & $19^{\circ} 6^{\prime} 57.95^{\prime \prime} \mathrm{N}$ & $155^{\circ} 48^{\prime} 53.02^{\prime \prime} \mathrm{W}$ & 666 & 1,344 & 12 & Volcanic \\
\hline Ka'ūpūlehu (H3) & $19^{\circ} 46^{\prime} 6.30^{\prime \prime} \mathrm{N}$ & $155^{\circ} 56^{\prime} 11.18^{\prime \prime} \mathrm{W}$ & 589 & 1,180 & 16 & Volcanic \\
\hline Honouliuli (H4) & $21^{\circ} 25^{\prime} 3.92^{\prime \prime} \mathrm{N}$ & $158^{\circ} 5^{\prime} 47.98^{\prime \prime} W$ & 740 & 2,911 & 19 & Volcanic \\
\hline Kaluakauila (H5) & $21^{\circ} 32^{\prime} 50.18^{\prime \prime} \mathrm{N}$ & $158^{\circ} 13^{\prime} 23.81^{\prime \prime} W$ & 448 & 1,738 & 32 & Volcanic \\
\hline Kamiloloa (H6) & $21^{\circ} 6^{\prime} 45.25^{\prime \prime} \mathrm{N}$ & $156^{\circ} 57^{\prime} 32.65^{\prime \prime} \mathrm{W}$ & 633 & 1,455 & 44 & Volcanic \\
\hline Kānepu'u 1 (H7) & $20^{\circ} 52^{\prime} 33.30^{\prime \prime} \mathrm{N}$ & $156^{\circ} 58^{\prime} 35.59^{\prime \prime} \mathrm{W}$ & 519 & 716 & 59 & Volcanic \\
\hline Kānepu'u 2 (H8) & $20^{\circ} 52^{\prime} 24.41^{\prime \prime} \mathrm{N}$ & $156^{\circ} 58^{\prime} 2.36^{\prime \prime} W$ & 515 & 709 & 59 & Volcanic \\
\hline Kānepu'u 3 (H9) & $20^{\circ} 52^{\prime} 41.00^{\prime \prime} \mathrm{N}$ & $156^{\circ} 59^{\prime} 18.45^{\prime \prime} \mathrm{W}$ & 523 & 712 & 58 & Volcanic \\
\hline Kanaio (H10) & $20^{\circ} 37^{\prime} 12.96^{\prime \prime} \mathrm{N}$ & $156^{\circ} 20^{\prime} 20.75^{\prime \prime} \mathrm{W}$ & 634 & 835 & 55 & Volcanic \\
\hline Lìhau (H11) & $20^{\circ} 51^{\prime} 29.87^{\prime \prime} \mathrm{N}$ & $156^{\circ} 35^{\prime} 50.27^{\prime \prime} W$ & 732 & 2,157 & 29 & Volcanic \\
\hline Waimea (H12) & $22^{\circ} 3^{\prime} 39.60^{\prime \prime} \mathrm{N}$ & $159^{\circ} 38^{\prime} 45.38^{\prime \prime} W$ & 320 & 1,799 & 31 & Volcanic \\
\hline Kōke'e 1 (H13) & $22^{\circ} 8^{\prime} 29.28^{\prime \prime} \mathrm{N}$ & $159^{\circ} 41^{\prime} 23.05^{\prime \prime} \mathrm{W}$ & 805 & 1,807 & 53 & Volcanic \\
\hline Kōke'e 2 (H14) & $22^{\circ} 8^{\prime} 51.98^{\prime \prime} \mathrm{N}$ & $159^{\circ} 40^{\prime} 4.77^{\prime \prime} \mathrm{W}$ & 1,014 & 1,915 & 58 & Volcanic \\
\hline Miloli'i (H15) & $22^{\circ} 7^{\prime} 36.32^{\prime \prime} \mathrm{N}$ & $159^{\circ} 41^{\prime} 2.05^{\prime \prime} \mathrm{W}$ & 944 & 1,755 & 52 & Volcanic \\
\hline
\end{tabular}

on incidence (Chao 2) using EstimateS software (Colwell 1997). Floristic similarities of native species, genera, and families were examined for all regions using PRIMER 5 (Clarke and Gorley 2001) by producing BrayCurtis dissimilarity matrices using all taxa after square-root-transforming the total basal area for each taxon per site to down weigh the impact of the most dominant taxa. Basal area was used to create the matrices because it provides an indication of the ecological dominance (Mueller-Dombois and Ellenberg 
TABLE 2

Largest Families from 400.1 ha Samples of All Woody Plants $\geq 2.5 \mathrm{~cm}$ dbh (Native and Nonnative) in Dry Forests of the Pacific

\begin{tabular}{|c|c|c|c|c|c|c|}
\hline Family & New Caledonia & Fiji & Marquesas & Marianas & Hawai' ${ }^{\circ} \mathrm{i}$ & Total \\
\hline Rubiaceae & 9 & 10 & 3 & 4 & 8 & 28 \\
\hline Euphorbiaceae & 11 & 9 & 1 & 1 & 5 & 25 \\
\hline Fabaceae & 7 & 13 & 1 & 5 & 4 & 23 \\
\hline Sapindaceae & 14 & 2 & 1 & - & 2 & 18 \\
\hline Myrtaceae & 10 & 2 & 2 & 1 & 5 & 17 \\
\hline Apocynaceae & 6 & 7 & 1 & 3 & 1 & 15 \\
\hline Rutaceae & 10 & 1 & - & - & 3 & 13 \\
\hline Ebenaceae & 5 & 3 & - & - & 2 & 10 \\
\hline Moraceae & 4 & 4 & 1 & 2 & 1 & 9 \\
\hline Rhamnaceae & 3 & 4 & - & 1 & 2 & 9 \\
\hline Thymelaeaceae & 2 & 2 & - & - & 5 & 9 \\
\hline Celastraceae & 3 & 2 & 1 & 1 & - & 7 \\
\hline Sapotaceae & 3 & 2 & - & 1 & 1 & 7 \\
\hline Flacourtiaceae & 3 & 2 & 1 & - & - & 6 \\
\hline Meliaceae & 2 & 2 & - & 1 & 1 & 6 \\
\hline Myrsinaceae & 1 & 3 & - & - & 2 & 6 \\
\hline Verbenaceae & 3 & 2 & 1 & 2 & 1 & 5 \\
\hline Araliaceae & 3 & - & - & - & 2 & 5 \\
\hline Oleaceae & 3 & - & - & 1 & 1 & 5 \\
\hline Agavaceae & - & 1 & - & - & 4 & 4 \\
\hline Anacardiaceae & 3 & 1 & - & - & 1 & 4 \\
\hline Malvaceae & - & 1 & 2 & 1 & 1 & 3 \\
\hline Nyctaginaceae & 1 & 1 & 1 & 1 & 1 & 3 \\
\hline
\end{tabular}

Note: Boldface indicates most speciose family by region.

2002). The resulting dissimilarity matrix was then used as the input for nonmetric multidimensional scaling to produce an ordination diagram based on 100 restarts. In addition, we calculated the percentage of native species, genera, and families shared among the five regions and with Australia (Fensham 1995). Incidence and percentages of native species in different categories of phenology (evergreen, semideciduous, deciduous), sexual systems (perfect, dioecious, monoecious), and dispersal type (anemochory, autochory, zoochory) were calculated for all native plants identified to species within each Pacific region.

\section{RESULTS}

\section{Family Composition}

A total of 299 species or morphospecies was identified in 40 dry forest sites in the Pacific: 267 taxa were identified to species, 16 plants were identified to genus, 11 plants were identified to family, and five plants could not be identified to family. A total of 57 families was identified from all sites in the Pacific: 54 of the families are native to the Pacific and three families were nonnative to the region (Bignoniaceae, Caricaceae, Pinaceae). Rubiaceae (28 spp.), Euphorbiaceae (25 spp.) Fabaceae (23 spp.), Sapindaceae (18 spp.), and Myrtaceae (17 spp.) were the most speciose families in Pacific dry forests. New Caledonia had the highest number of families (39) followed by Fiji (36), Hawai'i (33), the Marianas (22), and the Marquesas (13). The most frequently speciose dry forest families in the Pacific were Rubiaceae, Fabaceae, Euphorbiaceae, and Myrtaceae, which were all in the top five most speciose families in three regions (Table 2). However, no family clearly dominated across regions.

\section{Species Composition}

Dry forest species with the highest frequency and density within each region provide an overview of the dominant floristic composition of Pacific dry forests (Table 3). New 
TABLE 3

Floristic Composition of the 10 Most Frequent and Abundant Woody Plants $\geq 2.5 \mathrm{~cm}$ dbh from 0.1 ha Samples of Dry Forests in the Pacific

\begin{tabular}{|c|c|c|c|c|}
\hline Region & Family & Scientific Name & Frequency & Density \\
\hline \multirow{10}{*}{$\begin{array}{l}\text { New Caledonia } \\
(n=7)\end{array}$} & Moraceae & Malaisia scandens ${ }^{\mathrm{L}}$ & 6 & 106 \\
\hline & Verbenaceae & Premna serratifolia & 6 & 29 \\
\hline & Euphorbiaceae & Croton insularis & 5 & 101 \\
\hline & Euphorbiaceae & Drypetes deplanchei & 5 & 22 \\
\hline & Rhamnaceae & Rhamnella vitiensis ${ }^{\mathrm{L}}$ & 5 & 17 \\
\hline & Fabaceae & Acacia spirorbis & 4 & 272 \\
\hline & Rubiaceae & Gardenia urvillei & 4 & 203 \\
\hline & Euphorbiaceae & Codiaeum peltatum & 4 & 187 \\
\hline & Meliaceae & Dysoxylum bijugum & 4 & 54 \\
\hline & Sapindaceae & Cupaniopsis trigonocarpa & 4 & 51 \\
\hline \multirow{10}{*}{$\begin{array}{l}\text { Fiji } \\
(n=9)\end{array}$} & Sapotaceae & Planchonella grayana & 8 & 22 \\
\hline & Euphorbiaceae & Mallotus tilifolius & 7 & 140 \\
\hline & Hernandiaceae & Gryrocarpus americanus & 7 & 69 \\
\hline & Verbenaceae & Premna protrusa & 7 & 26 \\
\hline & Moraceae & Malaisia scandens ${ }^{\mathrm{L}}$ & 6 & 140 \\
\hline & Rubiaceae & Psydrax odorata & 6 & 39 \\
\hline & Fabaceae & Mucuna gigantea ${ }^{\mathrm{L}}$ & 6 & 29 \\
\hline & Rubiaceae & Antirbea inconspicua & 6 & 23 \\
\hline & Fabaceae & Derris trifoliata & 6 & 19 \\
\hline & Ebenaceae & Diospyros phlebodes & 5 & 262 \\
\hline \multirow{10}{*}{$\begin{array}{l}\text { Marquesas } \\
(n=6)\end{array}$} & Euphorbiaceae & Xylosma suaveolens & 6 & 206 \\
\hline & Apocynaceae & Cerbera manghas & 5 & 242 \\
\hline & Celastraceae & Maytenus crenata ${ }^{\mathrm{L}}$ & 5 & 80 \\
\hline & Moraceae & Ficus prolix & 5 & 14 \\
\hline & Sapindaceae & Sapindus saponaria & 4 & 188 \\
\hline & Myrtaceae & Eugenia reinwardtiana & 4 & 155 \\
\hline & Malvaceae & Thespesia populnea & 4 & 76 \\
\hline & Rubiaceae & Morinda citrifolia & 4 & 17 \\
\hline & Euphorbiaceae & Glochidion marchionicum & 3 & 22 \\
\hline & Verbenaceae & Premna serratifolia & 3 & 5 \\
\hline \multirow{10}{*}{$\begin{array}{l}\text { Marianas } \\
(n=3)\end{array}$} & Fabaceae & Cynometra ramiflora & 3 & 498 \\
\hline & Annonaceae & Guamia mariannae & 3 & 214 \\
\hline & Oleaceae & fasminum marianum & 3 & 78 \\
\hline & Rubiaceae & Psychotria mariana & 3 & 27 \\
\hline & Fabaceae & Erythrina variegata & 3 & 15 \\
\hline & Moraceae & Ficus tinctoria & 3 & 15 \\
\hline & Rubiaceae & Dendrocnide latifolia & 3 & 9 \\
\hline & Apocynaceae & Alyxia torresiana & 3 & 5 \\
\hline & Fabaceae & Leucaena leucocephala* & 2 & 65 \\
\hline & Apocynaceae & Neisosperma oppositifolia & 2 & 54 \\
\hline \multirow{10}{*}{$\begin{array}{l}\text { Hawaiian Islands } \\
(n=15)\end{array}$} & Ebenaceae & Diospyros sandwicensis & 10 & 569 \\
\hline & Oleaceae & Nestegis sandwicensis & 8 & 326 \\
\hline & Fabaceae & Leucaena leucocephala* & 6 & 148 \\
\hline & Anacardiaceae & Schinus terebinthifolius* & 6 & 96 \\
\hline & Myrtaceae & Metrosideros polymorpha & 5 & 202 \\
\hline & Rubiaceae & Psydrax odorata & 5 & 113 \\
\hline & Sapindaceae & Dodonaea viscosa & 5 & 103 \\
\hline & Euphorbiaceae & Aleurites moluccana* & 5 & 78 \\
\hline & Myrtaceae & Psidium guajava* & 5 & 29 \\
\hline & Sapotaceae & Pouteria sandwicensis & 4 & 17 \\
\hline
\end{tabular}

Note: Frequency, number of Gentry transects the species is found in; density, total number of stems within all Gentry transects of the region; ${ }^{\mathrm{L}}$, liana; ${ }^{*}$, nonnative. 


\section{TABLE 4}

Native Species Richness and Chao 2 Estimates of Native Species Richness, Percentage of Native Species Sampled, and Lower and Upper Bounds of Native Species Richness Based on 95\% Confidence Intervals (CI)

\begin{tabular}{|c|c|c|c|c|c|}
\hline Region & $\begin{array}{c}\text { Observed } \\
\text { Species Richness }\end{array}$ & $\begin{array}{c}\text { Estimated } \\
\text { Species Richness }\end{array}$ & $\begin{array}{c}\text { Estimated } \\
\text { Percentage of } \\
\text { Species Sampled }\end{array}$ & $\begin{array}{c}95 \% \text { CI } \\
\text { Lower Bound }\end{array}$ & $\begin{array}{c}95 \% \text { CI } \\
\text { Upper Bound }\end{array}$ \\
\hline New Caledonia & 118 & 178 & $66 \%$ & 149 & 231 \\
\hline Fiji & 72 & 105 & $68 \%$ & 86 & 150 \\
\hline Marquesas & 12 & 12 & $97 \%$ & 12 & 19 \\
\hline Marianas & 26 & 32 & $81 \%$ & 28 & 51 \\
\hline Hawai'i & 52 & 81 & $64 \%$ & 64 & 125 \\
\hline
\end{tabular}

Caledonia had a total of 131 species from seven sites. Three tree (Premna serratifolia L., Croton insularis Baill., Drypetes deplanchei (Brongn. \& Gris) Merr.) and two liana species (Malaisia scandens (Lour.) Planch., Rbamnella vitiensis (Benth.) A. C. Sm.) were encountered at over $70 \%$ of the dry forest sites. Fiji had a total of 89 species from nine sites. Four trees (Planchonella grayana H. St. John, Mallotus tilifolius (Blume) Mūll. Arg., Gyrocarpus americanus Jacq., Premna protrusa A. C. Sm. \& S. P. Darwin) were found in over $70 \%$ of the dry forests. The Marquesas contained 17 species from six sites. Four species occurred in over $70 \%$ of the sites (Xylosma suaveolens (J. R. Forst. \& G. Forst.) G. Forst., Cerbera manghas L., Maytenus crenata (G. Forst.) Lobr.-Callen, Ficus prolixa G. Forst.). There were 34 species encountered from three sites in the Marianas. Eight species were encountered at all three sites. There were 66 species encountered at 15 sites in Hawai' 1 . Two tree species (Diospyros sandwicensis (A. DC.) Fosberg, Nestegis sandwicensis (A. Gray) O. Deg., I. Deg. \& L. A. S. Johnson) were encountered at over $50 \%$ of the sites. The dry forests of Hawai' $\mathrm{i}$ contain a high incidence and density of nonnative species.

\section{Native Species Richness}

New Caledonia and Fiji contained the highest observed native species richness followed by Hawai'i, the Marianas, and the Marquesas (Table 4). Levels of native species richness within a region were similar to Chao 2 estimates of total species richness, with New Caledonia estimated to contain the highest estimated species richness and the Marquesas containing the lowest. Native dry forest species were best sampled in the Marquesas and the Marianas; however, our samples from New Caledonia, Fiji, and Hawai'i appear to have sampled only between $64 \%$ and $68 \%$ of all native dry forest trees, shrubs, and lianas.

\section{Floristic Similarity}

Floristic similarities of native species, genera, and families were examined using percentage similarities among all regions for the three taxonomic levels (Table 5). There is very low similarity in composition at the native species level between the Pacific regions, with no more than 10 species being shared between any of the regions. A few shared species may account for a considerable percentage (up to $42 \%$ [Table 5]) in the depauperate dry forest floras of the Marianas and Marquesas, with 26 and 12 native species, respectively. Several of the species shared between the Marianas and Marquesas and other regions are also widespread coastal species, such as Erythrina variegata L., Hibiscus tiliaceus L., and Premna serratifolia, that may occasionally be found in inland dry forests.

We also illustrated relationships among study sites for all regions using nonmetric multidimensional scaling (NMDS) from a Bray-Curtis dissimilarity matrix. The NMDS had low to moderate stress values (these indicate how well the two-dimensional NMDS plots correspond to the dissimilarity matrix) of 0.09 at the species level, 0.19 at the generic level, and 0.18 at the family level (Figure 2). At the species level, Hawai'i has the lowest 
TABLE 5

Pairwise Percentage Similarities (Taxa Shared between Two Regions/(Sum of Taxa in Region A and Region $\mathrm{B} / 2) \times 100)$ at the Native Species/Genus/Family Level between Regions

\begin{tabular}{lccrrr}
\hline \hline & Australia & New Caledonia & Fiji & Marquesas & Marianas \\
\hline New Caledonia & $7(10) / 38 / 71$ & & & & \\
Fiji & $5(6) / 28 / 66$ & $7(7) / 41 / 75$ & & & \\
Marquesas & $1(1) / 8 / 30$ & $5(3) / 14 / 43$ & $10(4) / 24 / 49$ & & \\
Marianas & $0(0) / 17 / 43$ & $1(1) / 19 / 55$ & $8(4) / 28 / 63$ & $26(5) / 34 / 58$ & \\
Hawai'i & $1(1) / 15 / 51$ & $2(2) / 19 / 57$ & $2(1) / 18 / 58$ & $3(1) / 13 / 44$ & $0(0) / 20 / 53$ \\
\hline
\end{tabular}

Note: Total number of species/genera/families for each region: Australia, 174/120/49; New Caledonia, 118/90/35; Fiji, 71/63/34; the Marquesas, 12/12/11; the Marianas, 26/23/20; Hawai' $\mathrm{i}$, 50/36/25. Number of species shared is in parentheses.

degree of similarity, and New Caledonia, Fiji, and the Marquesas have the highest clustering. In addition, all sites within a region cluster more or less closely together at that level. At the genus level, some Fijian and New Caledonian sites are placed in close proximity on the NMDS plot, indicating a high similarity between the two regions. At the family level, sites do not cluster clearly according to region, except in the Marquesas and some sites in Hawai' $\mathrm{i}$. This shows that the composition of families among dry forests of the Pacific is relatively similar.

\section{Natural History Characteristics}

The natural history characteristics of native species were relatively similar among regions (Table 6). Tropical dry forests in the Pacific have a high proportion of evergreen species and a low number and proportion of semideciduous and deciduous species. Sexual systems throughout the Pacific averaged $62 \%$ perfect, $19 \%$ dioecious, and 19\% monoecious flowers. The proportion of dioecious species was highest in Hawai' $i$, and the proportion of monoecious species was highest in the Marquesas. Zoochory was the most common dispersal type in the Pacific, followed by autochory and anemochory.

\section{DISCUSSION}

\section{Tropical Dry Forests of the Pacific}

Tropical dry forest or seasonally dry tropical forest have been loosely defined as forest in frost-free regions with 500-2,000 $\mathrm{mm}$ of an- nual precipitation and a pronounced dry season of 4 to 7 months (Holdridge et al. 1971, Walter 1971, Murphy and Lugo 1986) or more conservatively defined as occurring in frost-free regions with $<1,800 \mathrm{~mm}$ of annual precipitation and a dry period of at least 5 to 6 months with $<100 \mathrm{~mm}$ precipitation each month (Gentry 1995, Pennington et al. 2009). Native dry forests in New Caledonia and the Marquesas clearly fall within both these classifications, as do a majority of sites in Fiji and Hawai'i. However, dry forests in the Marianas receive high annual precipitation, and there were three sites in Fiji, two sites in Hawai'i, and one site in the Marianas that receive over $2,000 \mathrm{~mm}$ of annual precipitation according to WorldClim. This suggests that our results in these regions contain gradients from dry to more moist or mesic native forest sites on the dry sides of these regions.

\section{Family Composition}

The family compositions of the Pacific are relatively similar to those of the neotropical dry forests with the exception of Bignoniaceae, in which native species in the Pacific are almost completely absent. Neotropical forests from 0.1 ha samples are dominated by Fabaceae, Bignoniaceae, Rubiaceae, Sapindaceae, Euphorbiaceae, Capparidaceae, Flacourtiaceae, Myrtaceae, and Apocynaceae (Gentry 1995). However, unlike dry forests of the neotropics, no families in the Pacific dominated within or across regions. It appears that Fabaceae, Rubiaceae, Sapindaceae, Euphorbiaceae, Myrtaceae, and Apocynaceae families are the 

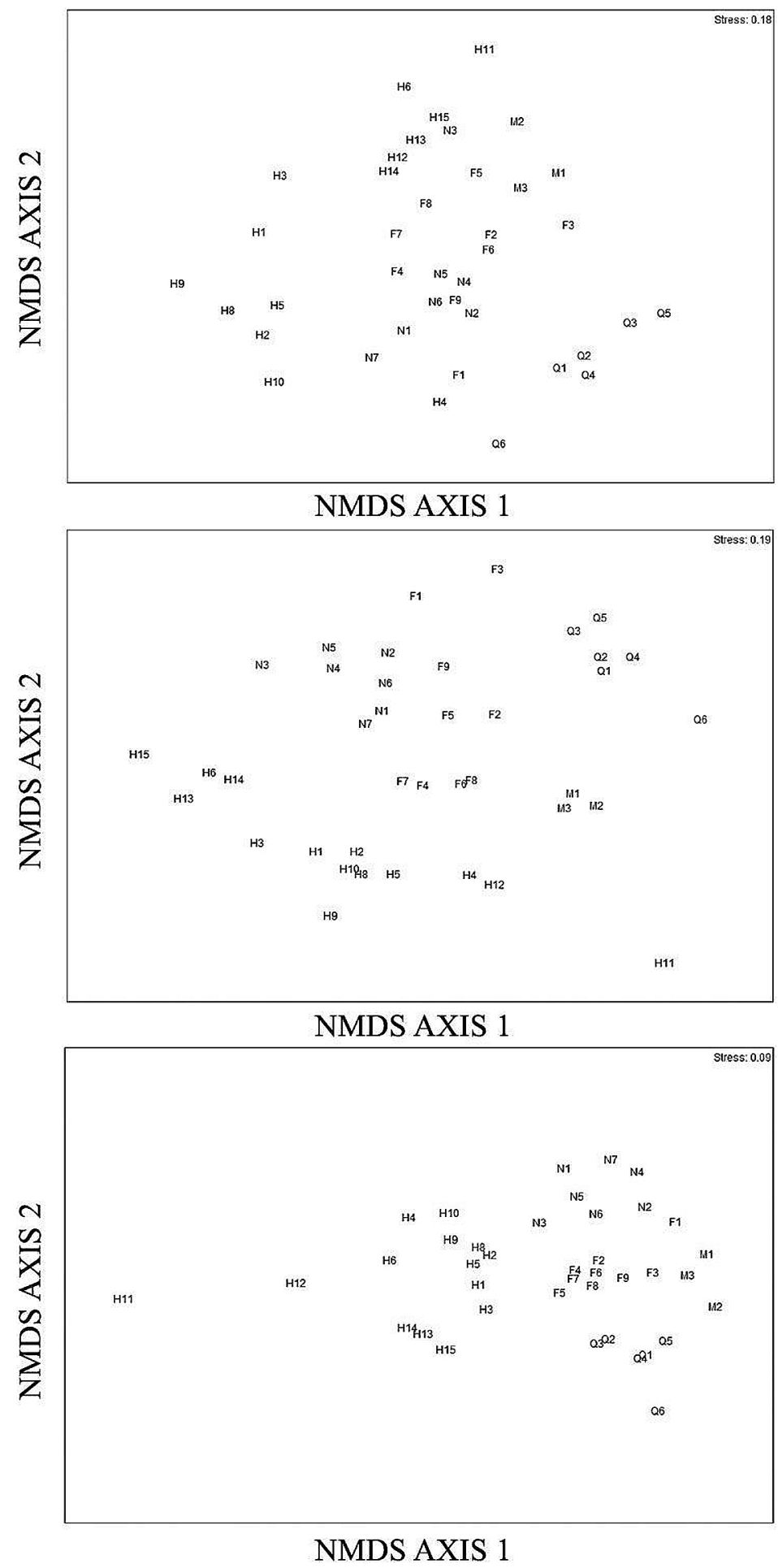

FIgURE 2. Nonmetric multidimensional scaling (NMDS) plots constructed from Bray-Curtis dissimilarity matrices based on the square-root-transformed basal areas of native species (top), genera (middle), and families (bottom) in Gentry transects in the 40 study sites. Study sites correspond to site code in Table 1: Hawai' $\mathrm{i}, \mathrm{H}$; New Caledonia, N; Fiji, F; Marianas, M; Marquesas, Q. 


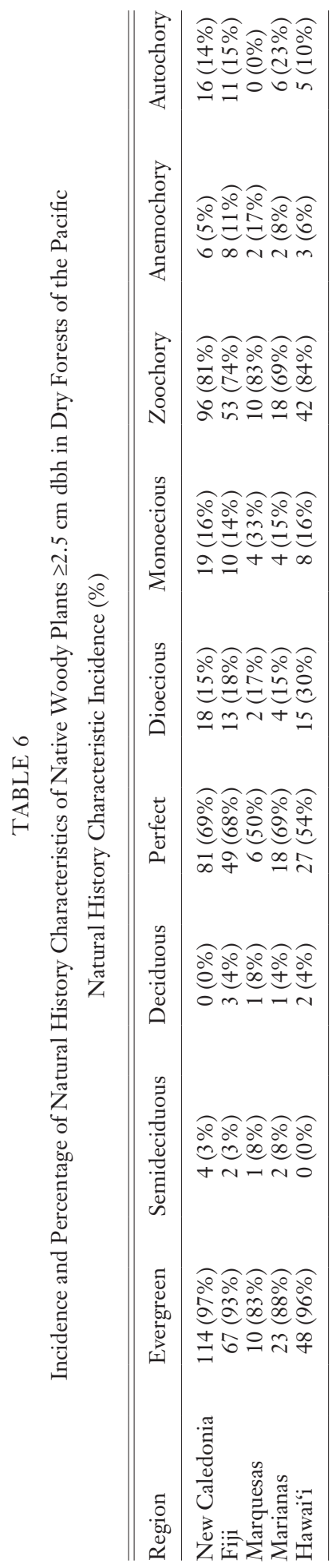

largest families of woody plants in Pacific and neotropical dry forests.

\section{Species Composition}

Although there are several native genera that are widespread in Pacific dry forests, such as Ficus, Diospyros, Maytenus, Pisonia, Premna, Alyxia, and Psychotria, there is extremely low similarity among native dry forest species in the Pacific. This is similar to the high species turnover reported among neotropical dry forest fragments (Pennington et al. 2009) and not unexpected because of the long distances (thousands of kilometers) between the regions. Psydrax odorata (G. Forst.) A. C. Sm. \& S. P. Darwin was encountered in four regions (New Caledonia, Fiji, the Marquesas, Hawai'i), three species were encountered in three regions, and 16 species were encountered in two regions. There are a number of studies that suggest that a majority of the tree and liana species in the Pacific have colonized from Malesian (Malay Peninsula, Indonesia, Philippines, New Guinea) and Australia, and this appears to be true with dry forest taxa (Wagner et al. 1990, Jaffré et al. 1993, Fensham 1995, Meyer 2004, Jaffré et al. 2008, Keppel et al. 2009). There were only three native pantropical species that occur in both the Pacific and the neotropics (Gyrocarpus americana, Dodonaea viscosa Jacq., Mucuna gigantea (Willd.) DC.) (Gentry 1995).

\section{Native Species Richness}

Observed and estimated levels of native species richness clearly suggest that New Caledonia and Fiji contain the highest species richness in Pacific dry forests, followed by Hawai'i, the Marianas, and the Marquesas. Gentry's transect method appears to be appropriate for quantifying patterns of species richness and floristic composition in regions where little comparative field data exist. Indeed, $48 \%$ of all 109 known native dry forest trees and shrubs of Hawai' $i$ were encountered in 15 sites that covered a total of 1.5 ha (Pau et al. 2009). This is relatively similar to the estimated percentage of species sampled in Hawai'i (64\%) and within the lower and 
upper bounds of total native species estimated (64 and 125 species, respectively) in the region based on $95 \%$ confidence intervals.

\section{Floristic Similarity}

Despite the low number of native species shared, several of the dominant trees of dry forests in New Caledonia, Fiji, and the Marquesas are widely distributed throughout the South Pacific. Although New Caledonia dry forest has high endemism (59\%), many of the dominant species, such as Croton insularis, Drypetes deplanchii, and Malaisia scandens, can also be found in dry forests of Australia, with most of the rare endemic species restricted to a few sites that are currently more open and disturbed (Jaffré et al. 1993, Fensham 1995, Jaffré et al. 2008). The dry side of Fiji and the Marquesas appear to be dominated by widely distributed trees, such as Cerbera manghas, Gyrocarpus americanus, and Premna serratifolia, that also occur in drier areas of the Pacific and Australia (Fensham 1995, Franklin et al. 2006). Some of these trees also commonly occur in coastal beach vegetation and are dispersed by ocean currents (Ghazanfar et al. 2001), likely facilitating their wide distribution. The dry forests of Hawai' $i$ are composed of a high number of endemic species with $90 \%$ endemism (Pau et al. 2009), and our field data suggest that Hawaiian dry forests contain a high frequency and density of nonnative trees compared with other dry forests in the Pacific. Dry forests in Hawai' $i$ have been classified into six types dominated by endemic species in the genera Diospyros, Nestegis, Acacia, Erythrina, Metrosideros, and Sapindus (Gagné and Cuddihy 1990). In accordance with this, our study sites were dominated by the same native endemic species.

\section{Natural History Characteristics}

Tropical dry forests of the Pacific have different phenology and dispersal types compared with continental neotropical dry forests. There were only seven deciduous species in the Pacific. The leaf phenology in the Pacific appears different from that in mainland neo- tropical dry forests, where a majority of species lose their leaves in the dry season (Holdridge et al. 1971, Bullock 1985, Gentry 1995). Results are more similar to those from dry forests in the Caribbean, which contain few deciduous species (Lugo et al. 2006). The phenology pattern of dry forest on islands is possibly due to a pronounced maritime influence on islands that results in less intensive dry seasons compared with extreme dry periods in the continental neotropics.

Hawaiian dry forests have a high proportion of dioecious species (28\%), and this is consistent with the entire Hawaiian flora, in which $32 \%$ of species are dioecious (Sakai et al. 1995, Pau et al. 2009). Results from sexual systems in native tropical trees in Hawai' $i$ (perfect 54\%, dioecious 30\%, monoecious $16 \%)$ are relatively similar to those in dry forests in Florida (perfect $60 \%$, dioecious $28 \%$, monoecious 12\%) (Gillespie 2006), Nicaragua/Costa Rica (perfect 63\%, dioecious 22\%, monoecious 15\%) (Gillespie 1999), and Chamela, Mexico (perfect 58\%, dioecious 26\%, monoecious 16\%) (Bullock 1985). However, dry forests on other Pacific islands contain a lower proportion of dioecious species (range 15\% to $18 \%$ ), which suggests that high dioecy may not be as common on oceanic islands when compared with the mainland (Whittaker and Fernández-Palacios 2007).

Wind dispersal has been identified as a selective disadvantage on oceanic islands in the tropics, and this appears true for dry forests of the Pacific (Carlquist 1974). Tropical dry forest trees of Central America are composed of approximately $32 \%$ anemochory (winddispersed seeds), and tropical dry forests in South America are composed of approximately 26\% anemochory (Gentry 1995, Gillespie 1999). However, dry forests in the Pacific contain a lower number and proportion of wind-dispersed species.

\section{CONCLUSIONS}

The dry forests of the Pacific have a family composition relatively similar to that of neotropical dry forests; however, there is no consistently dominant family of trees within 
Pacific dry forests. New Caledonia and Fiji contain the highest native species richness followed by Hawai'i, the Marianas, and the Marquesas. There is very little overlap at the species level among regions, with Hawaiian dry forests the most dissimilar at the native species, genus, and family level and New Caledonia and Fiji the most similar. Unlike mainland neotropical dry forest, dry forests in the Pacific contain very few deciduous species and a low proportion of wind-dispersed species. There is a high proportion of dioecious species in Hawai'i, which is similar to the neotropics; however, other Pacific regions have fewer dioecious species.

\section{ACKNOWLEDGMENTS}

We thank Christophe Lambert (Secrétariat general de la Province Sud, Nouméa) for permission to work in New Caledonia, and Gilles Dagostini and Jerôme Munzinger for help with plant identification at the IRD Herbarium at Nouméa. We thank Christine Farris, Victoria Michelle, and Danielle Ellis for collecting field data in New Caledonia. We thank the National Trust of Fiji and especially local villages and chiefs for permission to do field research in Fiji. Craig Morly, Peter Harlow, Pita Biciloa, and Clare Morrison provided technical support, and Robert Gillespie, Kathy Gillespie, Suzanne Morrison, and Carolina Sanchez provided assistance in field research. Laura L. Williams from the Division of Fish and Wildlife was extremely helpful in the Mariana Islands. We thank the federal and state agencies and the Nature Conservancy of Hawai' $i$ for granting us permission to do research in the Hawaiian Islands. We thank Kapua Kawelo and Dan Sailer for transportation and technical support on O'ahu. We thank Clyde Imada and Napua Harbottle at the Bishop Museum for permission to examine herbarium specimens. We thank Elizabeth Del Cid, Jo Sito, Scott Pipken, Markie Daniel, Robert Gillespie, Kathy Gillespie, Glen MacDonald, Irem Sepil, Creighton Leigh, and John May for assistance with field surveys in Hawai'i. Two anonymous reviewers provided excellent suggestions and comments that greatly improved the manuscript.

\section{Literature Cited}

Bawa, K. S., D. R. Perry, and J. H. Beach. 1985. Reproductive biology of tropical lowland rain forest trees. I. Sexual systems and incompatibility mechanisms. Am. J. Bot. 72:331-345.

Bullock, S. H. 1985. Breeding systems in the flora of a tropical deciduous forest in Mexico. Biotropica 17:287-301.

Carlquist, S. 1974. Island life. The Natural History Press, New York.

Clarke, K. R., and R. N. Gorley. 2001. PRIMER v5. user manual/tutorial. Plymouth routines in multivariate ecological research. Plymouth Marine Laboratories, Plymouth, UK.

Colwell, R. K. 1997. EstimateS. v8.2. University of Connecticut, Storrs.

Craig, R. J. 1992. Ecological characteristics of a native limestone forest of Saipan, Marianas Islands. Micronesica 25:85-97.

Fensham, R. 1995. Floristics and environmental relations of inland dry rainforest in north Queensland, Australia. J. Biogeogr. 22:1047-1063.

Franklin, J., S. Wiser, D. R. Drake, L. Burrows, and W. R. Sykes. 2006. Environment, disturbance history, and rain forest composition across the islands of Tonga, western Polynesia. J. Veg. Sci. 17:233-244.

Gagné, W. C., and L. W. Cuddihy. 1990. Vegetation. Pages 45-114 in W. L. Wagner, D. R. Herbst, and S. H. Sohmer, eds. Manual of the flowering plants of Hawai' $i$. University of Hawai'i Press, Bishop Museum Press, Honolulu.

Gentry, A. H. 1982. Patterns of Neotropical plant species diversity. Evol. Biol. 15:1-84. . 1988. Changes in plant community diversity and floristic composition on environmental and geographical gradients. Ann. Mo. Bot. Gard. 75:1-34.

$$
\text { 1995. Diversity and floristic compo- }
$$
sition of Neotropical dry forests. Pages 146-194 in S. H. Bullock, H. A. Mooney, and E. Medina, eds. Seasonally dry tropical forests. Cambridge University Press, Cambridge.

Ghazanfar, S. A., G. Keppel, and S. Khan. 2001. Coastal vegetation of small islands 
near Viti Levu and Ovalau, Fiji. N. Z. J. Bot. 39:587-600.

Gillespie, T. W. 1999. Life history characteristics and rarity of woody plants in tropical dry forest fragments of Central America. J. Trop. Ecol. 15:637-649.

- 2006. Diversity, biogeography, and conservation of woody plants in tropical dry forest of Florida. Pages 383-394 in R. T. Pennington, G. P. Lewis, and J. A. Ratter, eds. Neotropical savannas and seasonally dry forests: Plant diversity, biogeography, and conservation. CRC Taylor and Francis Group, Boca Raton, Florida.

Gillespie, T. W., and T. Jaffré. 2003. Tropical dry forests of New Caledonia. Biodivers. Conserv. 12:1687-1697.

Harlow, P. S., and P. N. Biciloa. 2001. Abundance of the Fijian crested iguana (Brachylophus vitiensis) on two islands. Biol. Conserv. 98:223-231.

Hatheway, W. H. 1952. Composition of certain native dry forests: Mokuleia, Oahu. Ecol. Monogr. 22:153-168.

Hijmans, R. J., S. E. Cameron, J. L. Parra, P. G. Jones, and A. Jarvis. 2005. Very high resolution interpolated climate surfaces for global land areas. Int. J. Clim. 25:1965-1978.

Holdridge, L. R., W. C. Grenke, W. H. Hatheway, T. Liang, and J. A. Tosi. 1971. Forest environments in tropical life zones: A pilot study. Pergamon Press, Oxford.

Jaffré, T., P. Morat, and J. M. Veillon. 1993. Etude floristique et phytogéographique de la forêt sclérophylle de NouvelleCalédonie. Bull. Mus. Hist. Nat. Bot. Ser. 15:107-147.

Jaffré, T., F. Rigault, and J. Munzinger. 2008. Identification and characterization of floristic groups in dry forests of relicts of a west coast region of New Caledonia. Pac. Conserv. Biol. 14:128-145.

Janzen, D. H. 1988. Tropical dry forests: The most endangered major tropical ecosystem. Pages 130-137 in E. O. Wilson, ed. Biodiversity. Natural Academy Press, Washington, D.C.

Keppel, G. 2006. The lowland rain forest of the Solomon Islands: A bleak future. Mel. Geogr. 3:14-17.
Keppel, G., Y. M. Buckley, and H. P. Possingham. 2010. Drivers of lowland rain forest community assembly, species diversity and forest structure on islands in the tropical South Pacific. J. Ecol. 98:87-95.

Keppel, G., A. J. Lowe, and H. P. Possingham. 2009. Changing perspectives on the biogeography of the tropical South Pacific: Colonisation, dispersal, extinction, speciation and vicariance. J. Biogeogr. 36: 1035-1054.

Keppel, G., and M. V. Tuiwawa. 2007. Dry zone forests of Fiji: Species composition, life history traits, and conservation. N. Z. J. Bot. 45:545-563.

Lerdau, M., J. Whitbeck, and N. M. Holbrook. 1991. Tropical deciduous forest: Death of a biome. Trends Ecol. Evol. 6:201-202.

Lugo, A. E., E. Medina, J. C. Trejos-Torres, and E. Helmer. 2006. Botanical and ecological basis for the resilience of Antillean dry forests. Pages 359-381 in R. T. Pennington, G. P. Lewis, and J. A. Ratter, eds. Neotropical savannas and seasonally dry forests: Plant diversity, biogeography, and conservation. CRC Taylor and Francis Group, Boca Raton, Florida.

Meyer, J.-Y. 2004. Threat of invasive alien plants to native flora and forest vegetation of eastern Polynesia. Pac. Sci. 58:357-375.

- 2007. Conservation des forêts naturelles et gestion des aires protégées en Polynésie française. Bois For. Trop. 291:25-40.

Mueller-Dombois, D., and H. Ellenberg. 2002. Aims and methods of vegetation ecology. Blackburn Press, Caldwell, New Jersey.

Murphy, P. G., and A. E. Lugo. 1986. Ecology of tropical dry forest. Annu. Rev. Ecol. Syst. 17:67-88.

Myers, N., R. A. Mittermeier, C. G. Mittermeier, G. A. da Fonseca, and J. Kent. 2000. Biodiversity hotspots for conservation priorities. Nature (Lond.) 403:853-858.

Pau, S., T. W. Gillespie, and J. P. Price. 2009. Natural history, biogeography, and endangerment of Hawaiian dry forest trees. Biodivers. Conserv. 18:3167-3182. 
Pennington, R. T., M. Lavin, and A. OliveiraFilho. 2009. Woody plant diversity, evolution, and ecology in the tropics: Perspectives from seasonally dry tropical forests. Annu. Rev. Ecol. Evol. Syst. 40:437457.

Rolett, B., and J. Diamond. 2004. Environmental predictors of pre-European deforestation on Pacific islands. Nature (Lond.) 431:443-446.

Sakai, A. K., W. L. Wagner, D. M. Ferguson, and D. R. Herbst. 1995. Origins of dioecy in the Hawaiian flora. Ecology 76:2517-2529.

Stone, B. C. 1970. The flora of Guam. Micronesica 6:1-659.
Wagner, W. L., D. R. Herbst, and S. H. Sohmer.1990. Manual of the flowering plants of Hawai'i. University of Hawai'i Press, Bishop Museum Press, Honolulu.

Wagner, W. L., and D. H. Lorence. 2003. Flora of the Marquesas. Smithsonian Institution and National Tropical Botanical Garden (http://botany.si.edu/ pacificislandbiodiversity/marquesasflora/ intro.htm). Accessed 27 October 2006.

Walter, H. 1971. Ecology of tropical and subtropical vegetation. Oliver and Boyd, Edinburgh.

Whittaker, R. J., and J. M. FernándezPalacios. 2007. Island biogeography: Ecology, evolution, and conservation. Oxford University Press, Oxford. 
